framum

Sociológico

\section{Forum Sociológico}

Série II

33 | 2018

Número 33

\title{
Disputa identitária em torno do conceito de autenticidade : Uma análise a partir do caso da cultura bretã (França)
}

Dispute identitaire autour du concept d'authenticité: Une analyse à partir du cas de la culture traditionnelle bretonne (France)

Identity dispute around the concept of authenticity: An analysis based on the case of traditional Breton culture (France)

\section{Luciana de Araujo Aguiar}

\section{OpenEdition}

Journals

Édition électronique

URL : https://journals.openedition.org/sociologico/2432

DOI : $10.4000 /$ sociologico. 2432

ISSN : 2182-7427

Éditeur

CICS.NOVA - Centro Interdisciplinar de Ciências Sociais da Universidade Nova de Lisboa

Édition imprimée

Pagination : $37-46$

ISSN : 0872-8380

\section{Référence électronique}

Luciana de Araujo Aguiar, « Disputa identitária em torno do conceito de autenticidade : Uma análise a partir do caso da cultura bretã (França) », Forum Sociológico [En ligne], 33 | 2018, mis en ligne le 30 décembre 2018, consulté le 30 mars 2022. URL : http://journals.openedition.org/sociologico/2432 ; DOI : https://doi.org/10.4000/sociologico.2432 


\title{
DISPUTE IDENTITAIRE AUTOUR DU CONCEPT D'AUTHENTICITÉ: UNE ANALYSE À PARTIR DU CAS DE LA CULTURE TRADITIONNELLE BRETONNE (FRANCE)
}

\author{
DISPUTA IDENTITÁRIA EM TORNO DO CONCEITO DE AUTENTICIDADE: UMA ANÁLISE \\ A PARTIR DO CASO DA CULTURA BRETÃ (FRANÇA) \\ IDENTITY DISPUTE AROUND THE CONCEPT OF AUTHENTICITY: AN ANALYSIS BASED \\ ON THE CASE OF TRADITIONAL BRETON CULTURE (FRANCE)
}

\section{Luciana de Araujo Aguiar}

LERSEM - Laboratoire d'Études et de Recherches en Sociologie et en Ethnologie de Montpellier, Université Paul Valery - Montpellier 3, França

\begin{abstract}
Résumé
Dans cet article je souhaite comprendre les positionnements des acteurs insérés dans l'univers de la culture traditionnelle bretonne et leur prise de position par rapport à l'authenticité des aspects culturels bretons. Dans le milieu de la culture traditionnelle bretonne il y a une variété d'acteurs qui existent et coexistent avec des dispositions différenciées. Ces acteurs se positionnement par référence à l'authenticité des origines et l'inauthenticité d'une exploitation commerciale ou médiatique de leurs pratiques. Les disputes autour de l'authenticité des pratiques culturelles bretonnes font sens pour tous les acteurs, qui ont tous à se définir par rapport à elle pour légitimer les rapports entre eux. Cet article montrera les discours d'authentification de la culture bretonne, et tout en particulier, les représentations autour des pratiques d'authenticité à propos de la musique bretonne. À partir d'une recherche à la fois ethnographique et théorique, j'envisage d'analyser les disputes identitaires autour du concept $d$ >authenticité à partir de l'analyse de la culture traditionnelle bretonne.
\end{abstract}

Mots-clés: authenticité, culture traditionnelle bretonne, champ culturel

\section{Resumo}

Neste artigo procuramos compreender os posicionamentos dos atores inseridos no universo da cultura tradicional bretã e as suas tomadas de posição sobre a autenticidade dos aspectos culturais bretões. Neste universo, coexistem uma variedade de atores com diferentes disposições. Esses atores posicionam-se por referência à autenticidade das origens e à inautenticidade de uma exploração comercial ou mediática das suas práticas. As disputas sobre a autenticidade das práticas culturais bretãs fazem sentido para todos os atores, que se definem em relação a esta autenticidade para legitimar as relações entre si. Este artigo mostrará os discursos de autentificação da cultura bretã e, em particular, as representações em torno das práticas de autenticidade a propósito da música bretã. Com base em uma pesquisa etnográfica e teórica, pretendo analisar as disputas identitárias em torno do conceito de autenticidade a partir da análise da cultura tradicional bretã.

Palavras-chave: autenticidade, cultura tradicional bretã, campo cultural 


\begin{abstract}
In this article I try to understand the positioning of actors in the world of traditional Breton culture and their position on the authenticity of Breton cultural aspects. In this universe, a variety of actors with different dispositions coexists. These actors position themselves by reference to the authenticity of their origins and the inauthenticity of a commercial or media exploitation of their practices. Disputes over the authenticity of Breton cultural practices make sense for all actors, who define themselves in relation to this authenticity to legitimize relations among each other. This article will show the speeches of authenticity of the Breton culture and in particular the representations around the practices of authenticity of Breton music. Based on an ethnographic and theoretical research, I intend to analyze the identity disputes around the concept of authenticity from the analysis of traditional Breton culture.
\end{abstract}

Keywords: authenticity, traditional Breton culture, cultural field

\section{Introduction}

Dans cet article je souhaite comprendre les positionnements des acteurs insérés dans le champ de la culture traditionnelle bretonne et leur prise de position par rapport à l'authenticité des aspects culturels bretons. Il y a une variété d'acteurs qui existent et coexistent dans ce milieu culturel avec des dispositions différenciées. Ces acteurs se positionnement par référence à l'authenticité des origines et l'inauthenticité d'une exploitation commerciale ou médiatique de leurs pratiques. Les disputes autour de l'authenticité des pratiques culturelles bretonnes font sens pour tous les acteurs, qui ont tous à se définir par rapport à elle pour légitimer leurs rapports entre eux.

Les acteurs mettent en place des stratégies, conscientes ou la plupart du temps inconscientes, pour confirmer la plus grande authenticité de leurs pratiques culturelles. La quête de l'authenticité est généralement revendiquée en prenant appui sur des principes d'originalité et de singularité collectivement reconnus. Les acteurs mettent en œuvre le discours de légitimation expérientielle, généralement associé à des arguments historiques et géographiques, comme pratique d'authentification. Ils justifient I'authenticité par le fait que leurs ancêtres, établis Bretagne ont éprouvé ces pratiques culturelles par le passé et les leur ont transmis (directement ou indirectement). Ils seraient, ainsi, les héritiers d'une filiation culturelle authentique.

Les notions de stratégie et d'authenticité sont mobilisées comme fils rouges de cette réflexion, ainsi que celles de champ, position, prise de position, disputes symboliques et stratégies. Je porte ainsi une attention particulière aux concepts développés par Pierre Bourdieu. Le cadre théorique bourdieusien m'est apparu comme pertinent pour analyser le milieu de la culture traditionnelle bretonne, constitué d'individus occupant des positions différenciées - structurées principalement par des degrés d'authenticité manifeste - et se mettant en relation au cours de disputes symboliques, dans le but de légitimer la traditionnalité de leurs représentations dans leurs pratiques culturelles.

L'objectif visé par cet article est, donc, de comprendre les positionnements des acteurs et leur prise de position par rapport à l'authenticité de la culture bretonne: les différences, les prises de distance, les effets de rupture, les avant-gardismes et les conservatismes. A partir d'une analyse à la fois ethnographique et théorique, j'envisage d'analyser les disputes identitaires autour du concept d'authenticité à partir de l'analyse de la culture traditionnelle bretonne.

L'article bénéficie du travail de terrain réalisé en 2014 , pour ma thèse ${ }^{1}$ de doctorat en Ethnologie soutenue en décembre 2017 à l'Université Paul-Valéry (France). Ayant comme l'un des principaux intérêts pour ma thèse les bals de fest-noz ${ }^{2}$, je me suis rendue en Bretagne pour une immersion d'environ deux mois en 2014. Mon but était d'observer différents bals de fest-noz dans plusieurs départements et de m'entretenir avec des personnes issues de différentes zones de la région et ayant des opinions distinctes sur cette expression culturelle. De ce fait, j'ai rencontré des personnes très fières des fest-noz et qui s'identifiaient à la Bretagne par ses pratiques culturelles, ainsi que d'autres individus qui ne reconnaissaient pas le fest-noz comme marqueur de l'identité bretonne. J'ai été amenée à côtoyer les pratiquants de fest-noz en grande partie grâce au groupe Fest-noz and traditional musics in Brittany du réseau social couchsurfing $^{3}$, et par les cercles celtiques ${ }^{4}$ présents dans la majorité des villes de Bretagne. Ayant un financement de I'université Paul-Valéry pour bien à mener le travail sur le terrain, j'ai effectué mes recherches dans trois des quatre départements de la Bretagne. En Ille-et-Vilaine, j'ai rencontré des gens lors de bals à Rennes et Saint-Malo; dans les Côtes-d'Armor, j'ai participé à des fest-noz à Perros Guirrec, en faisant connaissance avec les gens de la ville; et dans le Finistère, j'ai pu m'entretenir 
avec des gens lors des bals de Quimper, Brest, Landerneau, Plouzané ou encore Collorec.

\section{Le champ de la culture traditionnelle bretonne}

La notion de champ me parait comme pertinente pour analyser le milieu de la culture traditionnelle bretonne, constitué par des acteurs et des groupes qui, même s'ils occupent des positions opposées, s'accordent sur la lutte pour l'affirmation de l'authenticité de la culture bretonne. Les discours d'authentification de la culture bretonne sont à la fois la condition et le produit du fonctionnement de ce milieu culturel. Le fait que leur identité soit constituée par la culture bretonne apparait comme une évidence aux acteurs et groupes qui sont engagés dans cet espace. Cependant, tous ne considèrent pas comme authentiques les mêmes aspects de cette culture bretonne. De ce fait, s'ils s'unissent autour de discours généraux affirmant l'existence et la valeur d'une identité bretonne, ils n'attribuent pas tous une authenticité aux mêmes pratiques culturelles. Le champ de la culture traditionnelle bretonne est ainsi structuré par des positions différenciées sur l'authenticité, elles-mêmes relatives à une plus ou moins grande appropriation de capital en jeu dans cet univers: le capital social (fruit des rapports constitués avec d'autres agents et des réseaux sociaux qu'ils mobilisent).

Le concept de champ désigne un espace positionnel formé par un système de positions qui détermine la forme des interactions et qui organise un espace de coups possibles. D'après Bourdieu:

Le champ est un réseau de relations objectives (de domination ou de subordination, de complémentarité ou d'antagonisme, etc.), entre des positions (...) chaque position est objectivement définie par sa relation objective aux autres positions, ou, en d'autres termes, par le système des propriétés pertinentes, c'est-à-dire efficientes, qui permettent de la situer par rapport à toutes les autres dans la structure de la distribution globale des propriétés. (Bourdieu, 1992, p. 321)

Un champ désigne ainsi un univers spécialisé possédant un circuit de disputes entre acteurs par des positions de pouvoir, établies de façon relationnelle à partir du capital en dispute dans un champ donné, ce qui suppose des règles propres et spécifiques qui délimitent les disputes et déterminent les hiérarchies.

D'après Dirkx (s/d), le champ désigne toute partie de l'espace social ayant acquis un degré d'autonomie suffisant pour reproduire elle-même la croyance dans le bien-fondé de son principe fondateur. Autrement dit, conditionnée par une indépendance relative envers les contraintes externes (religieuses, politiques, économiques, médiatiques), l'autonomie d'un champ est sa capacité interne à se doter soi-même d'un principe de différenciation et d'auto-organisation (Dirkx, s/d). L'espace social est une autre notion clé dans la théorie de Bourdieu. L'espace social est une représentation abstraite d'un ensemble de positions coexistant dans un même monde social et définies les unes par rapport aux autres par des relations de proximité, de voisinage et d'éloignement (Bourdieu, 1994). L'espace social est ainsi structuré selon une logique de la différence, cela veut dire que les positions prises par les agents se définissent en s'opposant à celles prises par les autres, en se distinguant des autres. Ce vaste ensemble se construit comme une structure de positions différenciées qui se définissent par la place qu'elles occupent dans la distribution d'une espèce particulière de capital.

Le concept d'espace social forgé par P. Bourdieu vise ainsi à appréhender le monde social de manière relationnelle. Chaque individu occupe une position sociale qui a une certaine existence et une certaine stabilité uniquement parce qu'elle est relativement différente de celle d'autres individus ou d'autres groupes. Dans ce schéma, les individus et groupes sociaux ont une position d'autant plus élevée qu'ils détiennent un grand volume de capital. La position des individus dans l'espace social dépend donc du volume et du type de capital détenu par chacun. L'opposition entre l'authentique et le simili, présente dans la structure des deux espaces sociaux étudiés, oriente les positionnements des agents et leur engagement dans la défense de l'authentique, qui est d'autant plus efficace s'ils possèdent une plus grande quantité de l'espèce particulière de capital en jeu. F. Plomb (2003) remarque qu'au-delà du volume de capital (toutes espèces de capital confondues) qui donne une idée de la position hiérarchique des individus, $P$. Bourdieu met l'accent sur la différenciation entre les différents types de capital possédés pour placer les individus et les groupes dans l'espace social.

Les notions d'espace social et champ insistent toutes les deux sur la dimension relationnelle d'un milieu, sur les traits qui n'existent que dans et par la relation avec d'autres propriétés Néanmoins, le concept de champ est plus spécialisé que le concept d'espace social. En effet, le champ désigne une partie de l'espace social ayant acquis un degré d'autonomie importante. L'espace social serait ainsi "découpé" en un certain nombre de champs et de sous-champs dans lesquels s'affrontent les agents sociaux disposant de divers capitaux en quantité et en qualité inégales selon leur position dans ce même espace social. Les deux concepts sont à la fois appropriés et adaptés pour la discussion mise en œuvre dans cet article, néanmoins, comme le milieu de la culture traditionnelle bretonne se réfère à un 
univers relativement autonome, j'utiliserai plutôt le terme "champ de la culture traditionnelle bretonne" au cours de cet article.

\section{Les acteurs du champ de la culture traditionnelle bretonne}

Les acteurs du champ de la culture traditionnelle bretonne possèdent un discours politique de défense d'une identité manifestée dans les musiques, danses, chants et instruments traditionnels. Les aspects culturels bretons émergent comme des potentiels symboliques porteurs d'une tradition singulière. La prise de conscience d'un héritage culturel est le déclencheur d'actions visant la reconnaissance érudite et institutionnelle et la mise en patrimoine de ces pratiques culturelles. L'agentivité des acteurs transforme les traditions en ressources identitaire, économique et touristique et joue un rôle fondamental dans la production de la "vraie" culture bretonne. Mais qui sont-ils ces acteurs?

Le champ de la culture traditionnelle bretonne bretonne révèle un univers hétérogène et multiple. Dès mes premiers jours en Bretagne, j'ai compris qu'il s'agissait d'un terrain extrêmement divers et j'ai donc décidé d'explorer cette diversité à travers mes interlocuteurs. Les dispositions qu'ils ont acquises au cours de leur trajectoire nous aident à comprendre la raison de leur investissement dans cet espace de relations. Les personnes avec lesquelles j'ai établi des rapports ont entre 16 et 80 ans. Ils sont enseignants (souvent de disciplines liées à I'univers breton comme la langue, la musique et la danse), ouvriers, étudiants, professionnels libéraux, salariés agricoles, techniciens, professionnels du spectacle ou de la culture, retraités et stars régionales (célèbres chanteurs de kan ha diskan ou de breton/ gallo, rockstars ou fameux musiciens mélangeant la musique bretonne à d'autres genres).

Parmi les acteurs côtoyés pendant le travail sur le terrain, il mérite d'être mentionné Maurice Poulmarc'h et Ronan Guéblez, célèbres chanteurs de kan ha diskan'; Alan Pierre, animateur d'ateliers de danses bretonnes et conseiller technique en danse bretonne à la Fédération de danse bretonne War'l Leur Penn Ar Bed; Glenn Jegou, créateur d'un des plus grands festivals de musique bretonne: le Festival Yaouank, réalisé annuellement à Rennes et directeur de la fédération culturelle Skeudenn Bro Roazhon qui rassemble cinquante-trois associations bretonnes ayant pour objectif de développer la culture bretonne dans le pays de Rennes ${ }^{6}$; Guy, clarinettiste dans un groupe de musique bretonne et enseignant retraité de musique au sein du cercle celtique de Rennes; Gurvan Molac, musicien, chanteur et auteur de plusieurs CDs de musique bretonne à danser; Gilbert le Pennec guitariste dans l'un des groupes de musique bretonne les plus connus dans le milieu de la "culture traditionnelle": le groupe Carré Manchot; Korentin et Camille, joueurs dans le groupe de musique War-Sav, formé par six jeunes garçons qui ont tous étudié au lycée Diwan7 Félix Le Dantec; Charles Quimbert, chanteur breton, directeur de Bretagne Culture Diversité, une plateforme associative subventionnée par le Conseil Régional pour traiter la question du patrimoine culturel immatériel en Bretagne et ex directeur de I'association Dastum, I'une des institutions culturelles les plus connues dans le domaine de la sauvegarde des pratiques culturelles bretonnes dites traditionnelles et responsable par la composition du dossier de patrimonialisation du fest-noz; Vincent Morel, conservateur animateur du patrimoine oral à Dastum et collecteur de chansons, contes et légendes auprès des "anciens" de la Haute-Bretagne; Karl, professeur de danse traditionnelle bretonne et responsable du cercle celtique Quic en Groigne de Saint-Malo; Yohann et Elie avides danseurs dans les bals bretons.

Les parcours des acteurs sont importants pour contextualiser leurs positions par rapport à l'authenticité des pratiques culturelles bretonnes. Leur trajectoire au sein de la région Bretagne influence leurs manières d'agir et leurs discours. La société bretonne s'installe en eux sous la forme de dispositions durables et de propensions structurées à penser, sentir et agir.

\footnotetext{
Chaque agent, qu'il le sache ou non, qu'il le veuille ou non, est producteur et reproducteur de sens objectif: parce que ses actions et ses œuvres sont le produit d'un modus operandi dont il n'est pas le producteur et dont il n'a pas la maîtrise consciente. (Bourdieu, 1972, p. 182)
}

Leurs actions et discours se trouvent donc socialement structurés et orientent leur investissement culturel au sein de cet espace de relations. Néanmoins, leurs actions et réponses créatives aux contraintes et aux exigences de leur environnement social, leur permettent d'agir activement dans le monde. Par exemple, Maurice Poulmarc'h, n'aurait pas été un célèbre chanteur de kan ha diskan s'il n'avait pas rencontré d'autres chanteurs à Paris, où il a aménagé pour trouver du travail. L'apprentissage du chant et de la langue bretonne au sein de sa famille pendant son enfance et adolescence a guidé postérieurement ses actions et son engagement dans la culture traditionnelle. Les relations qu'il a nouées à Paris ont été fondamentales dans son devenir de chanteur célèbre.

Les dispositions relatives à une origine sociale et géographique valorisée favorisent bien souvent des placements rentables symboliquement, et permettent de comprendre les positions que les agents occupent vis-à-vis de l'authenticité de la culture bretonne. Le fait d'être né dans le golfe du Morbihan et d'avoir 
écouté enfant les chansons bretonnes sur Radio-Armorique, ont guidé la trajectoire d'Alan Pierre au sein du cercle celtique de Vannes et en tant que conseiller technique en danse bretonne. De même, Glenn Jegou, Guy, Gurvan Molac, Ronan Guéblez et Korentin ont une position sociale rentable symboliquement, de par leurs origines géographiques au sein de la Région Bretagne, et de par leurs positions sociales héritées de familles ayant des attaches à cette culture. Leurs positions personnelles et professionnelles dominantes au sein des cercles celtiques (comme Guy), dans les institutions de promotion de la culture bretonne (comme Glenn Jegou) ou dans des groupes de chant et musique bretonne (comme Korentin, Gurvan Molac et Ronan Guéblez), ainsi que leurs discours sur l'authenticité de la culture bretonne sont le fruit de leurs dispositions, associées à leurs origines sociales et géographiques. Il y a également des agents qui n'ont pas une position très rentable symboliquement dans cet espace mais dont les trajectoires en faveur d'un engagement pour la culture bretonne ont été aussi déterminées par leurs origines géographiques et familiales (comme Elie et Yohann). Même si tous les acteurs cités dans ce paragraphe ont des dispositions associées à leurs origines géographiques et sociales, leurs trajectoires au sein de cercles celtiques, d'institutions de promotion de la culture bretonne ou de groupes de chant et musique bretonne, favorisent des placements plus ou moins rentables symboliquement. Au fil de leurs trajectoires, ils incorporent de nouvelles dispositions qui informent sur le placement qu'ils vont avoir au sein de cet espace.

Le système de dispositions, produit d'une trajectoire sociale, trouve tout au long de cette trajectoire des occasions plus ou moins favorables au placement stratégique. Ainsi, même des acteurs qui n'ont pas des dispositions héritées de leur lieu de naissance ou de leur famille, trouvent au cours de leurs trajectoires une occasion favorable à leur mobilité sociale. Karl, par exemple, est né à Mayenne dans une famille n'ayant aucune attache avec la culture bretonne et a longtemps habité à Paris. Néanmoins, il est professeur de danse traditionnelle bretonne et responsable du cercle celtique Quic en Groigne de Saint-Malo. Gilbert le Pennec et Camille sont aussi des agents qui, sans attache géographique ou familiale à la Bretagne, ont réussi à se placer dans cet espace social grâce au rock breton (Gilbert le Pennec) et à leur trajectoire au sein de l'école Diwan (Camille). D'autres acteurs, nés en Bretagne mais sans aucune attache familiale à ses pratiques culturelles, ont réussi à se placer grâce à leurs trajectoires au sein d'institutions de promotion de la culture bretonne, telles que le cercle celtique de Rennes dans le cas de Clément.

Les agents possèdent des origines sociales distinctes et appartiennent à différentes classes sociales, établies selon la composition de leur capital économique, social et culturel, accumulé tout au long de leurs trajectoires. On peut observer que les agents qui possèdent un capital social fort (fruit des rapports constitués avec d'autres agents et des réseaux sociaux qu'ils mobilisent) sont plus susceptibles d'avoir un capital culturel fort (établi par leurs positions au sein des institutions culturelles en Bretagne, et surtout, dans des institutions publiques) mais pas nécessairement un capital économique fort. En effet, le rang social et le pouvoir spécifique que les agents se voient assumer dans un espace particulier dépendent d'abord du capital spécifique qu'ils peuvent mobiliser. Or dans l'espace qui nous concerne, on peut dire que plus le capital social et culturel d'un agent est élevé - ce qui est ici relatif à une position élevée au sein d'une institution culturelle -, plus grandes sont ses chances de déterminer le contenu des discours sur la traditionalité et l'authenticité des pratiques culturelles bretonnes.

Ainsi, des acteurs comme Charles Quimbert, Vincent Morel, Glenn Jegou, Ronan Guéblez et Alan Pierre, étant donné leur fort capital social et culturel, possèdent plus que les autres les moyens d'authentifier la culture bretonne. Leurs positions au sein d'institutions culturelles fortes comme I'association Dastum, I'association Bretagne Culture Diversité, la Fédération de danse bretonne War'l Leur Penn Ar Bed et la Fédération d'associations du Pays de Rennes Skeudenn Bro Roazhon, attestent du volume du capital spécifique qu'ils détiennent. En effet, les intérêts qui motivent les acteurs et les moyens qu'ils peuvent mettre en œuvre pour les satisfaire dépendent de leur position dans l'espace social, c'est-à-dire de leur capital culturel et du pouvoir qu'il leur donne.

Les dispositions acquises par ces agents à travers leurs relations dans la Région Bretagne jouent un rôle dans leurs choix esthétiques. La traditionalité des expressions culturelles des paysans est l'une des croyances qui soutiennent cet espace de relations. Le goût pour le populaire et pour les expressions culturelles des classes populaires caractérise la nouvelle bourgeoisie insérée dans cet espace et engagée dans les pratiques culturelles bretonnes conçues comme traditionnelles. Souvent originaire de la classe moyenne urbaine, la nouvelle bourgeoise, pourvue de diplômes universitaires, se réapproprie des expressions culturelles populaires. La "culture du peuple" est éprouvée par celui qui est dans une relation de "proximité distante", qui est une dimension fondamentale de la relation de la nouvelle bourgeoisie aux classes populaires et à leurs traditions (Bourdieu, 1979). Le choix esthétique scénique se constitue souvent par opposition aux choix d'autres agents et groupes. Pour se différencier et se distinguer des Bretons non insérés dans le milieu de la "culture traditionnelle", les agents 
disposent de mécanismes esthétiques basés sur le goût pour le populaire, ou l'idée qu'ils s'en font. La prise de position esthétique constitue I'occasion d'éprouver et d'affirmer une position dans ce milieu culturel comme rang à tenir ou distance à maintenir. Le goût pour le folk, présent dans ce milieu depuis sa genèse, se combine aux dispositions de cette nouvelle bourgeoisie pour déterminer les stratégies et trajectoires des acteurs.

Présenter la position sociologique de ces acteurs est un instrument méthodologique important pour comprendre la raison de leur investissement, les moyens qu'ils détiennent pour investir et les chances qu'ils ont de réussir à faire accepter leurs discours sur la traditionalité des pratiques culturelles bretonnes. En outre, les dispositions actuelles des acteurs et les dispositions qu'ils ont précédemment acquises quant aux pratiques culturelles bretonnes sont importantes pour comprendre la façon dont ils se positionnent par rapport à leur authenticité. Les dispositions des acteurs influencent non seulement leurs positions par rapport à ce qu'ils considèrent comme authentique, mais aussi leurs stratégies de lutte pour I'authentique au sein des disputes symboliques. La façon dont les acteurs se positionnent actuellement vis-à-vis de l'authenticité des aspects culturels bretons dépend à la fois des dispositions acquises dans ce domaine au cours de leur vie, ainsi que des spécificités de l'espace de disputes en question. La variété d'acteurs possédant des dispositions distinctes résulte en des prises de position différentes vis-à-vis de l'authenticité de la culture bretonne.

\section{Positionnements et disputes par rapport à l'authenticité de la culture bretonne}

Les acteurs se positionnent dans ce champ culturel non seulement en faisant référence à leur fidélité à la tradition, à leur capacité à intégrer des emprunts à d'autres styles musicaux, mais aussi en opposant la "pureté" des origines et I"inauthenticité" d'une exploitation commerciale. Leurs stratégies pour la reconnaissance d'une légitimité influencent la manière dont ils se positionnent et leurs discours sur cette authenticité. Considérer les moyens employés à la légitimation de l'authenticité de la culture bretonne comme des stratégies a du sens dans la mesure où les individus tendent à défendre l'originalité et la traditionnalité de cette culture. La "stratégie" est une notion chère à $P$. Bourdieu, qui I'analyse comme une pratique utilisée par des acteurs, consciemment ou inconsciemment, afin d'augmenter leur capital et, corrélativement, à maintenir ou améliorer leur position dans la structure de rapports de force (1979). La stratégie recouvre ainsi peu à peu l'ensemble des moyens entrepris en vue d'une finalité. C'est une notion de réflexion intéressante parce qu'elle permet d'établir une connexion entre les différents choix et prises de position qui rythment la trajectoire d'un agent.

A Dewerpe a consacré un article à "La 'stratégie' chez Bourdieu" (1996). D'après l'auteur, P. Bourdieu analyse cette notion dans ses relations avec les notions connexes d'habitus et de capital. La stratégie est "I'expression d'un programme ni-ni, entre subjectivisme et objectivisme, entre rationalité des acteurs et structure" (Dewerpe, 1996, p. 14). L'auteur souligne le fait que la stratégie est un mécanisme utilisé par un agent pour tirer le maximum de profit. La stratégie apparaît ainsi comme un "modèle de comportement, un mode d'action ou de conduite, une pratique qui a des effets bénéfiques, voire optimaux, conçus en terme de 'profit'" (Dewerpe, 1996, p. 2). Néanmoins, ce calcul n'a pas besoin d'être délibérément choisi, il peut être d'autant plus efficace qu'il n'est pas intentionnel. En effet, dans "Questions de sociologie" (Bourdieu, 1980 , p. 119), P. Bourdieu remarque que la stratégie " $n$ 'est pas le calcul cynique, la recherche consciente de la maximisation du profit spécifique, mais une relation inconsciente entre un habitus et un champ". Les stratégies sont ainsi "des actions objectivement orientées par rapport à des fins qui peuvent n'être pas les fins subjectivement poursuivies" (Bourdieu, 1980 , p. 119). P. Bourdieu envisage aussi la stratégie comme l'attitude de l'agent investi dans le jeu social. Dans les prochains paragraphes, je présenterai le positionnement des acteurs par rapport à la musique bretonne et leurs stratégies d'exhibition de l'authenticité.

La perception selon laquelle la musique et la danse bretonne sont les aspects culturels les plus authentiques de partage collectif dans la région Bretagne marque le positionnement des acteurs dans cet univers. Néanmoins, la façon dont ils se manifestent par rapport à la transmission de la danse et de la musique, aux espaces dédiés à leur mise en place et à la formation musicale plus authentique varie beaucoup. La question de la prise de position est toujours la relation d'un agent à un espace particulier et ses réponses dans le jeu qui est instauré par l'espace social. En même temps, quand l'agent s'insère dans un espace social particulier, les dispositions qu'il a acquises antérieurement vont délimiter ses actions et discours. Il y a donc un double sens dans la prise position. Si d'une part les positionnements sont les actions et discours déployés par les agents au sein d'un espace social spécifique, d'autre part la prise de position d'un agent marque la façon dont il "voit" le monde, le hiérarchise et le classifie. La prise de position dépend aussi de la façon dont les différents capitaux sont accumulés, mis en œuvre et mis en valeur afin de favoriser cette prise de cette position.

La musique instrumentale et vocale bretonne est considérée comme l'un des traits les plus remarqua- 
bles dans la culture bretonne. Même si les acteurs reconnaissent la grande variété et richesse de ce matériau musical, ils se manifestent différemment par rapport à l'authenticité des instruments et des pratiques vocales. Certains acteurs perçoivent le chant - comme le kan ha diskan - comme la principale pratique culturelle d'identification avec la Bretagne. Le chant serait une pratique plus authentique parce qu'il contient la langue bretonne, comme le mentionne Alain:

Je trouve [le chant] complet, car en plus de la musique, il y a la langue bretonne. Même si je ne la parle pas très bien, j'apprends grâce aux chansons. Dans ma vie j'ai pu représenter divers chants, et à travers les chants, les personnes qui les avaient interprétés" (Entretien avec Alain, 22/07/2014)

Le chant doit être appris à travers la langue bretonne, contrairement à la musique qui est apprise par des méthodes partagées par un grand nombre (notamment les partitions). Maurice Poulmarc'h est un autre acteur qui s'identifie au chant en breton. Dans l'extrait suivant, il souligne que I'altération actuelle du chant est due au changement du processus d'apprentissage des codes culturels bretons:

L'altération qu'on retrouve dans le chant est due au changement de la transmission de la langue: la diffusion de la langue n'est plus la même. À mon avis, dans 20 ans on parlera breton de la même façon qu'on parle le français ${ }^{8}$. Heureusement que la langue écrite est encore enseignée. Certains disent qu'elle évolue, mais il est important de préserver I'accent. (Entretien avec Maurice Poulmarc'h, $22 / 07 / 2014)$

Le kan ha diskan, considéré par Alain et Maurice Poulmarc'h comme la pratique culturelle bretonne la plus authentique, est perçu de la même façon par de nombreux acteurs et groupes. Avoir un chanteur de kan ha diskan dans un groupe de musique est une façon de mettre en valeur la traditionalité de son groupe. Le groupe de musique bretonne Carré Manchot, qui mélange la sonorité des instruments bretons à d'autres rythmes et qui anime les bals de fest-noz depuis trente ans, a au sein du groupe un chanteur de kan ha diskan depuis 2013.

La bombarde ${ }^{9}$ et biniou ${ }^{10}$ (instruments musicaux bretons) tout comme le kan ha diskan sont perçus par de nombreux acteurs comme ce qui apporte le plus d'authenticité. Lors d'une conversation informelle avec un acteur nommé Elie, il m'a confié qu'il apprécie "le trad"11 original avec la bombarde et le biniou" (carnet de terrain, 23/07/2014). Alain, le grand-oncle d'Elie, partage également ce discours.
Selon lui, son groupe Re An Are participe souvent à des festoù-noz où il y a uniquement des chanteurs et des sonneurs"12: "De plus en plus d'ailleurs, car finalement on aime bien se retrouver entre gens qui aiment bien la tradition" (Entretien avec Alain, 22/07/2014).

Les événements culturels bretons qui rassemblent uniquement des chanteurs de kan ha diskan et des sonneurs de bombarde et de biniou, peuvent être réalisés avec le projet - personnel ou communautaire, conscient ou inconscient - de ne pas oublier les chansons anciennes en les gardant vivantes. La catégorie de tradition employée par Alain est ici appropriée par les acteurs en tant qu'outil discursif d'une identité bretonne. L'incorporation de cette catégorie dans les discours provoque la construction du traditionalisme comme projet (Weil, 1991). Qualifiant les musiques et chants recréés sur les mêmes bases que celles d'autrefois, la catégorie de traditionnel vient marquer des différenciations au sein de la musique bretonne: "Normalement, je filme les nouveaux groupes, les groupes un peu plus jeunes. J'ai aussi une amie qui fait le montage, mais elle est beaucoup plus traditionnelle, elle va filmer les chanteurs, les sonneurs traditionnels" (Entretien avec Yohann, 04/07/2014). Par des nombreux acteurs, la musique bretonne mélangée à des arrangements musicaux contemporains n'est pas considérée comme traditionnelle. A l'inverse, la musique bretonne composée par des instruments considérés historiquement bretons, tels que la bombarde et le biniou, est pensée comme traditionnelle.

La présence de sonneurs de biniou et de bombarde ainsi que de chanteurs de kan ha diskan caractérise pour une partie des acteurs les "origines historiques" de cet espace et sa traditionalité: "D'un point de vue de la Tradition, il n'y avait jusque-là [la "vague folk" des années 1970] que les chanteurs de kan ha diskan et les sonneurs de couple [le couple bombarde et biniou" (Entretien avec Melaine Favennec, in Jigourel, 2009, p. 39). Les sonneurs de couple de bombarde et biniou et les chanteurs de kan ha diskan sont devenus les symboles du "vrai traditionnel". Les éléments bombarde, biniou et kan ha diskan ont été conçus comme la base de I'identité collective et comme le cadre d'une définition immuable, spontanée et d'une certaine façon, sacrée, de la tradition. Nous savons toutefois que les traditions sont des catégories constitutives de représentations culturelles, construites et produites par des sujets (des personnes ou des groupes de personnes) à une époque donnée. Elles ne sont pas immuables mais bien au contraire modifiées et résignifiées au gré de l'histoire et des stratégies conscientes et inconscientes des acteurs. La façon dont Melaine Favennec s'exprime dévoile une naturalisation de la catégorie de "tradition", incorporée de façon réifiée. La "vraie tradition" ou la "Tradition" 
avec le T majuscule comme l'écrit Jigourel, ne serait représentée que par les chanteurs de kan ha diskan et les sonneurs de couple.

Le discours de légitimation de la tradition bretonne par les instruments traditionnels a été réifié dès que sont apparus les premiers musiciens mélangeant des instruments électriques à la musique bretonne. Un extrait d'entretien avec Alan Stivell ${ }^{13}$ (Kokel, 2011, p. 17), dans lequel il parle de la disparition du festnoz traditionnel le montre bien: "J'ai regretté, en même temps, la disparition du fest-noz traditionnel (seulement un style de danse, deux au maximum, kan ha diskan et couple biniou-bombarde uniquement) dont j'aurais souhaité le maintien". L'arrivée des instruments électriques fait prendre conscience aux acteurs d'une tradition qui existait avant. De ce fait, la façon dont la pratique culturelle était exécutée auparavant devient surévaluée et considérée comme traditionnelle. Les groupes de musique bretonne, créés par la "vague folk" des années 1970, ont collaboré à la production de la notion d'une "culture bretonne moderne" et en faisant cela, ils ont du même coup instauré la notion de "culture bretonne traditionnelle" pour faire référence à celle qui existait avant eux. Néanmoins, les "bals modernes" n'ont pas été développés de façon harmonieuse avec les sonneurs et chanteurs de kan ha diskan. Dans son ouvrage, T. Jigourel (2009, p. 41) relate les conflits entre les groupes de musique et les sonneurs et chanteurs au début des années 1970:

Dans I'œil du cyclone, la guitare faisait autant d'effet aux purs et durs du kan ha diskan que la boest an diaoul [accordéon diatonique] à un curé au début du XXe siècle. Soïg Sibéril ${ }^{14}$ se souvient qu'au milieu des seventies "il y avait toujours des endroits interdits aux groupes... Il fallait presque prendre le gilet pare-balles. Je me souviens d'avoir vu des gens nous jeter des pièces de vingt centimes".

Si l'arrivée des instruments électriques a provoqué une surévaluation de l'authenticité du biniou et de la bombarde, la présence de ces instruments a attiré des acteurs qui auparavant ne s'intéressaient pas à la culture bretonne. Ces acteurs se positionnent par identification aux emprunts à d'autres styles musicaux, comme Yohann: "Pour moi, il doit y avoir une variété musicale. J'aime bien notamment les groupes de musique plus jeunes, plus dynamiques" (Entretien avec Yohann, 04/07/2014). Glenn Jagou se reconnait également dans cette variété musicale: "La culture bretonne est de plus en plus mélangée à des signes et des styles de musique urbains tels que l'électro, le rock, le hip-hop, etc." (Entretien avec Glenn Jegou, 12/07/2014).

L'arrivée des instruments électriques caractérise la formation des groupes de musique bre- tonne. Ces formations musicales sont composées de 3,4,5 personnes voire plus, ce qui diffère de la formation de couple (formée par deux personnes: le joueur de la bombarde et le joueur du biniou). De plus, la musique jouée par les groupes mélange la sonorité traditionnelle bretonne de la bombarde et du biniou à d'autres instruments musicaux, ce qui n'existe pas dans la formation de couple. Les groupes de musique ont conquis une place valorisée et privilégiée auprès des acteurs, en occupant d'une certaine façon le statut des sonneurs, comme Alain I'explique: "Les sonneurs de couple ne sont plus très demandés, à part les meilleurs" (Entretien avec Alain, 22/07/2014). Les groupes ont des influences variées telles que le punk, le rock électrique, le ska, le reggae, le hip-hop et même la musique brésilienne, comme c'est le cas du groupe Boufam Caval dans lequel il y a un musicien brésilien qui joue de la percussion ${ }^{15}$. Ces musiciens et chanteurs sont des artistes célèbres dans cet espace de relations et même les Bretons qui ne sont pas insérés dans cet espace les connaissent. Ils sortent des albums et attirent les danseurs dans les bals de fest-noz où ils vont jouer. En fait, une particularité de cet espace social est la territorialisation de la musique bretonne. Il existe plusieurs groupes qui sont très connus en Bretagne mais pas du tout hors de la région.

Les groupes de musique, plus que les chanteurs et sonneurs, attirent de plus en plus de monde vers les événements culturels bretons. La formation de ces groupes aux styles variés apporte une nouvelle dimension à la tradition et de nouveaux chemins à partir desquels l'identité bretonne est réaffirmée. Le style du groupe, leurs instruments de musique et leur plus ou moins grande traditionalité exerce une influence sur les acteurs, qui prennent en compte le groupe qui va jouer dans leurs choix de bal. Voici une expérience de terrain qui le démontre.

Lors de mes derniers jours de terrain, je me suis rendue au fest-noz de la fête de I'huitre à Riecsur-Belon ${ }^{16}$, notamment pour voir le groupe Sonerien Du. J'étais accompagnée d'un interlocuteur breton nommé Arno, qui me l'avait recommandé car selon lui, il s'agit du meilleur groupe de musique bretonne. Quand le Sonerien Du a commencé à jouer, nous étions autour d'une table en train de manger des huitres en compagnie d'un couple breton et d'un couple allemand d'une soixantaine d'années. Quand le groupe a commencé à jouer, la première chose que la femme bretonne a dit fut: "Ça, ce n'est pas le fest-noz". J'étais également un peu surprise car la sonorité du groupe était différente de ce que j'avais écouté tout au long de mon travail de terrain. Je me suis tout d'abord demandé comment les gens pouvaient réussir à danser sur cette base musicale composée de clavier, batterie, basse et guitare. Puis après un moment, je me suis rendue compte que non seulement ils dansaient sur cette base mais que 
la plupart étaient là spécifiquement pour ce groupe et sa musique.

Bien que le Sonerien Du soit considéré comme un groupe à succès et ayant plusieurs fans partout en Bretagne, il existe certaines personnes qui se disent fières du fest-noz et qui n'aiment pas ce groupe "non traditionnel". C'est le cas d'Elie qui, quand je lui ai dit que je me rendais dans un fest-noz où jouait le Sonerien Du, m'a alertée sur la non-traditionalité de ce groupe. Certains fans du Sonerien Du, comme Arno et d'autres Bretons, ne se souciaient pas du supposé "manque de tradition" de ce groupe: ils participent au fest-noz parce qu'ils aiment le style de musique proposé, même si certains comme Arno ne dansent pas. Ces acteurs ne tiennent pas un discours sur la nécessité d'un fest-noz traditionnel, à l'inverse d'Elie par exemple.

La position d'Elie et d'Arno par rapport au groupe Sonerien Du révèle la façon dont les acteurs évaluent la traditionalité et l'authenticité des groupes de musique. Si le Sonerien Du est considéré par Elie comme non authentique parce que les instruments traditionnels tels que le biniou et la bombarde ont peu de place dans la sonorité de ce groupe, il est perçu par Arno comme authentique parce qu'il est composé seulement de musiciens bretons et parce qu'il démontre que la musique bretonne peut avoir autant de succès que la musique pop. Pour des gens comme Arno, connaitre la discographie d'un groupe et le suivre dans les bals de fest-noz partout dans la région est une manière de réaffirmer ses liens avec la Bretagne et de comprendre les pratiques traditionnelles de son territoire. Le refus de suivre un groupe peut également indiquer un regard spécifique sur les traditions bretonnes. La façon dont les acteurs évaluent la traditionalité des groupes de musique est relative à la position qu'ils occupent dans la structure de cet espace social. Les personnes qui s'inscrivent au cœur de cet espace ont une représentation de la traditionalité des pratiques bretonnes qui est différente des individus qui sont un peu en marge mais qui les fréquentent comme Arno. Selon lui, le groupe produit une bonne ambiance et cela lui suffit. Mais Elie, fier danseur des bals de fest-noz qu'il considère comme véritables représentants de la culture bretonne, ne juge pas ce groupe traditionnel.

Si les groupes de musique ont un rôle important au sein de cet espace, il y a des endroits où ces formations musicales, plurielles et variées, ne s'y forment pas. C'est le cas des petites communes de Haute Cornouaille et dans les campagnes. Dans ces villages, on considère que la tradition bretonne de la musique est celle des sonneurs et chanteurs locaux et celle des petits groupes qui n'ont pas une grande variété musicale. Il existe ainsi dans cet espace social une variété d'images de la tradition et de l'identification territoriale qui sont relatives aux endroits où elles sont fabriquées et aux personnes qui les fabriquent.

\section{Considérations finales}

Les différentes positions occupées par les acteurs à l'intérieur de cet espace de relations influencent la façon dont ils conçoivent I'authenticité de la culture bretonne. Certains acteurs comme Yohann et Arno, ainsi que les représentants d'associations culturelles habitant les grandes villes, comme Glenn Jegou, vont défendre l'authenticité de la musique bretonne par le mélange des instruments bretons traditionnels à d'autres styles musicaux. A l'inverse, Elie enseignant de langue bretonne, son oncle Alain joueur de bombarde, et Maurice Poulmarc'h chanteur de kan ha diskan, vont défendre l'authenticité de la musique par ses aspects les plus "purs". Les positions sont donc des emplacements stratégiques, des places à défendre et à conquérir dans un espace de luttes. Ces stratégies n'ont pas besoin d'être délibérément choisie et peuvent être d'autant plus efficaces qu'elles ne sont pas intentionnelles.

Il y a ainsi une altérité sociale et culturelle présente dans cet espace de relations. Même si les pratiques culturelles bretonnes sont considérées comme un code culturel d'interaction sociale, il existe des façons différenciées de les légitimer et de les défendre. Les productions symboliques attachées à ces pratiques sont ancrées dans les expériences spécifiques et mouvantes d'acteurs qui occupent des positions différentes. Le processus d'interprétation des pratiques culturelles est également relatif à la position que des groupes sociaux distincts possèdent au sein de la structure. Ainsi, acteurs et groupes s'affrontent pour légitimer certaines pratiques culturelles bretonnes comme les plus traditionnelles.

\section{Notas}

${ }_{1}$ Ma thèse intitulée "Les stratégies d'authenticité et les politiques de patrimoine culturel immatériel: une étude à partir de deux cas" a pour but de comprendre les stratégies de construction d'authenticité et les politiques de patrimoine culturel immatériel à partir de l'étude de deux pratiques culturelles reconnues actuellement comme patrimoine culturel immatériel: le fest-noz, présent dans les villes et campagnes de la région Bretagne, en France; et le jongo, présent au sein des villes installées dans la vallée du fleuve Paraíba do Sul, dans la région Sudeste du Brésil. La démarche comparative présentée dans cette thèse résulte d'une double étude de cas, d'une part les rapports entre le fest-noz et l'authenticité en Bretagne, et d'autre part le jongo et l'authenticité afro-brésilienne.

2 Le fest-noz c'est un bal animé par des musiques et chansons inspirées de la sonorité de la musique celtique. Le fest-noz a été inscrit en 2011 sur l'inventaire du patrimoine culturel immatériel de France par le ministère de la Culture et de la Communication. Cette pratique a également été présentée par l'État français auprès de I'UNESCO (Organisation des Nations Unies pour l'éducation, la science et la culture), dans le but d'être enregistrée comme un bien culturel immatériel 
de l'humanité. Les bals de fest-noz contribuent à la diffusion d'une idée de la culture bretonne basée sur les expressions culturelles traditionnelles et à la circulation de personnes intéressées par ces pratiques.

${ }^{3}$ Un réseau social dont le principal objectif est d'assurer un service d'hébergement temporaire et gratuit, de personne à personne. Les adhérents du réseau social peuvent promouvoir des événements "meetings" pour encourager leurs rencontres.

${ }^{4}$ Les cercles celtiques sont des associations qui essaient de promouvoir ce qu'elles appellent la "culture bretonne" par des spectacles artistiques qui mettent en avant les danses et musiques d'un terroir spécifique.

${ }^{5}$ Chanter en kan ha diskan consiste à chanter une mélodie à deux ou plusieurs personnes sans qu'il y ait la moindre interruption de son entre le soliste et le chœur. Un premier chanteur interprète une première phrase mélodique sur laquelle vient se greffer, lors des derniers temps, un second chanteur, lequel en reprend seul I'exécution. Puis le premier chanteur, qui s'était tu durant la reprise de la première phrase par son acolyte, intervient à nouveau lors de la fin de cette phrase pour se confondre avec son partenaire avant d'enchaîner seul la seconde phrase. Le résultat sonore est une chanson dont les couplets sont interprétés alternativement par un ou plusieurs chanteurs, mais dont la fin de chaque phrase est scandée par la totalité des exécutants, au nombre réduit généralement à deux ou trois personnes. Chaque chanteur, qu'il soit kaner (meneur) ou diskaner (répondeur) peut faire évoluer la mélodie au gré de son imagination, durant les passages où il est seul, pourvu qu'il retrouve son partenaire au bon moment et sur les bons appuis mélodiques (Defrance, 1991, p. 137).

${ }^{6}$ Les "pays" ont été établis entre 1995 et 1999 lors de la Loi n 95-115 du 4 février 1995 d'Orientation pour l'Aménagement et le Développement du Territoire complétée par la Loi no 99-533 du 25 juin 1999 d'Orientation de I'Aménagement durable du Territoire (LOADDT) ou "Loi Voynet" afin de créer une solidarité entre espaces ruraux et urbains et de constituer un territoire de projet. L'article 25 de la Loi Voynet définit un "pays" comme un territoire qui présente une cohésion géographique, culturelle, économique ou sociale, pouvant être reconnu à l'initiative de communes ou de leurs groupements comme ayant vocation à former un pays. Le pays de Rennes est l'un des 26 pays issus de la "Loi Voynet".

7 Les écoles Diwan sont des institutions scolaires qui existent partout en Bretagne et où est assuré l'enseignement du breton en filière bilingue et en enseignement optionnel.

${ }^{8}$ D'après lui, le breton est une langue plus "dure" tandis que le français est plus "souple". Donc, il pense que dans quelques années la langue bretonne empruntera la façon de parler de la langue française.

9 Instrument de musique à vent à anche double.

10 Cornemuse bretonne.

11 Abréviation commune sur mon terrain du mot "traditionnel".
12 En Bretagne, le sonneur (soner en breton, sonnou en gallo (langue gallèse) est un musicien jouant de la bombarde ou du biniou ainsi que, parfois, de la clarinette bretonne.

13 Alan Stivell est à la base du concept de Musique celtique et de sa diffusion auprès du grand public. Il fut dans les années 1970 celui qui, par la musique, fit revivre la culture bretonne. Véritable militant, Alan Stivell marqua cette époque où la vague folk déferlait à travers le monde, ce monde qu'il parcourut dans tous les sens avec sa célèbre harpe celtique. Si Alan Stivell a popularisé la musique bretonne et celtique modernes, il a été, dans le monde, un des précurseurs de plusieurs genres musicaux: folkrock ou ambient, et surtout world-music, dont il a été le théoricien dès son album Reflets en 1970. Données disponibles en ligne sur: http://www.rfimusique.com/ artiste/musiques-monde/alan-stivell/biographie et http:// www.alan-stivell.com/index.php?lang $=$ fr\&p=bio [consulté le 16 janvier 2015].

14 Guitariste breton et l'un des pionniers de l'implantation de la guitare en tant qu'instrument dans la musique bretonne traditionnelle.

15 J'ai connu le musicien Marcelo Costa et le groupe Boufam Caval au fest-deiz qui s'est déroulé à Rennes pendant le Festival Tombée de la Nuit.

16 Commune du département du Finistère.

\section{Références bibliographiques}

Bourdieu, P. (1972). Esquisse d'une théorie de la pratique. Genève: Droz.

Bourdieu, P. (1979). La distinction. Critique sociale du jugement. Paris: Éd. De Minuit.

Bourdieu, P. (1980). Questions de sociologie. Paris: Éditions de Minuit.

Bourdieu, P. (1992). Les règles de l'art. Paris: Seuil.

Bourdieu, P. (1994). Raisons pratiques: Sur la théorie de l'action. Paris: Seuil.

Defrance, Y. (1991). Le kan ha diskan. À propos d'une technique vocale en Basse-Bretagne. Cahiers d'ethnomusicologie, 4, 131-134. Disponible en: http://ethnomusicologie.revues.org/1582

Dirkx, P. (s/d). Champ. In A. Glinoer, et D. Saint-Amand (Dirs.), Le lexique socius. Disponible en ligne sur: http://ressources-socius.info/index.php/ lexique/21-lexique/37-champ

Dewerpe, A. (1996). La "stratégie" chez Pierre Bourdieu Enquête, (3), 53-68.

Jigourel, T. (2009). Festoù-noz, histoire et actualité d'une fête populaire. Romorantin: Editions CPE.

Kokel, J. L. (2011). Fest-noz: La découverte chavagne. Editions Chemin d'Images.

Plomb, F. (2003). Support de cours stratification sociale. Fribourg: Université de Fribourg.

Recebido a 30/09/2017. Aceite para publicação a 15/06/2018.

Luciana de Araujo Aguiar (aguiar_luciana@hotmail.com). Doctorante en Ethnologie. LERSEM, Laboratoire d'Études et de Recherches en Sociologie et en Ethnologie de Montpellier, Université Paul Valery - Montpellier 3. Route de Mende - 34199 MONTPELLIER CEDEX 5, France. 